1 Médico del Hospital Municipal de San Miguel en Ortopedia y Traumatología (ex Municipio de General Sarmiento, Pcia. de Bs. As.). Docente de la Facultad de Medicina - UNLAM Universidad Nacional de La Matanza, desde 2012. Profesor de la Materia Optativa: Medicina Social. E-mail: giglioprado@ hotmail.com

2 Dentista. Hace Doctorado para obtener el título de profesor en Salud Pública en el Instituto Juan Lazarte. E-mail: veronikgonzalez@yahoo.com

\section{AS POLÍTICAS PÚBLICAS NA FORMAÇÃO DA FORÇA DE TRABALHO NA ÁREA DA SAÚDE NA AMÉRICA LATINA: DESAFIOS E POSSIBILIDADES}

\author{
PUBLIC POLICIES FOR THE TRAINING OF \\ THE WORKFORCE IN THE AREA OF HEALTH \\ CARE IN LATIN AMERICA: CHALLENGES AND \\ OPPORTUNITIES
}

\author{
Giglio Prado $^{1}$ \\ Verónica González ${ }^{2}$
}

RESUMEN: El presente ensayo pretende hacer un recorrido histórico sobre las diferentes políticas públicas en el marco de la formación de la fuerza humana en salud contextualizado principalmente en América Latina, con una fuerte influencia del pensamiento médico-social y la salud colectiva. La primera consideración que atraviesa este ensayo es que la educación universitaria debe ser un bien público, un derecho humano y que debe ser garantizada por el Estado. Se entiende que es un campo complejo, ya que los procesos de formación de profesionales y trabajadores del campo sanitario forman parte de una arena de lucha de concepciones y prácticas con repercusiones en el curso de los acontecimientos y en la conformación de las correlaciones de fuerza en nuestros países. Para terminar, se reflexiona sobre la necesidad de introducir cambios sustantivos en las currículas de las Facultades de Medicina y otras profesiones del campo de la salud, y de esta manera contribuir al imperativo ético de completar los ciclos de construcción de ciudadanía, de contar con servicios nacionales integrales de salud financiados desde rentas generales, con participación y control popular, que brinden servicios de calidad, oportunos, resolutivos, humanizados, fuera de las crueles características del mercado. Democracia y salud deben tornarse sinónimos.

PALABRAS CLAVES: Políticas Públicas, Educación Médica, Derechos.

RESUMO: O presente ensaio pretende fazer um percurso histórico ao longo das diferentes políticas públicas, no marco da formação da força humana na saúde, contextualizado principalmente na América Latina, com marcada influência do pensamento médico-social e na saúde coletiva. A primeira consideração que permeia este ensaio é que a educação universitária deve ser um bem público, um direito humano, e deve ser garantida pelo Estado. Este é um campo complexo, já que os processos de 
formação de profissionais e trabalhadores do campo sanitário fazem parte de uma arena de luta de conceitos e práticas, com repercussões no decorrer dos acontecimentos e na conformação das correlações de força em nossos países. Para finalizar, reflete-se sobre a necessidade de introduzir mudanças substantivas nas grades curriculares das Faculdades de Medicina e outras profissões no campo da saúde, contribuindo deste modo com o imperativo ético de completar os ciclos de construção da cidadania, de contar com serviços nacionais integrais de saúde financiados com verbas gerais, com participação e controle popular, que ofereçam serviços de qualidade, oportunos, resolutivos, humanizados, fora das cruéis características do mercado. Democracia e saúde devem se tornar sinônimos.

PALAVRAS-CHAVE: Políticas Públicas; Educação Médica; Direitos.

ABSTRACT: This essay takes a historical look at the different public policies within the framework of the training of the workforce in the area of health care, contextualized mainly in Latin America, which is heavily influenced by medico-social thought and collective health. The first consideration that permeates this essay is that university education must be a public good, a human right, and as such, must be guaranteed by the State. This is a complex field, as the processes of training professionals and workers in the field of health care are part of an arena of struggle of concepts and practices, with repercussions on the course of events and on the conformation of the correlations of force in our Countries. Finally, we reflect on the need to introduce substantial changes in the curricula of medical schools and other professions in the field of health care, thereby contributing to the ethical imperative to complete the cycles of building citizenship, in order to have national services that are integrated with health, financed with general funds, with the participation and control of the people, and that provide quality, timely, resolutive, humanized services, outside the cruel characteristics of the market. Democracy and health must become synonymous.

KEYWORDS: Public Policies, Medical Education, Rights. 
1.- En primer lugar, quisiera AGRADECER LA INVITACIÓN al CONGRESO INTERNACIONAL SOBRE POLÍTICAS PÚBLICAS - ITAJAÍ, BRASIL. Explicitar que soy un médico ganado por el ideario de la Medicina Social Latinoamericana, ambiente al que he podido acceder por la generosa actitud de muchos compañeros y en cuyos quehaceres me he implicado. No soy un teórico de la educación médica, sí comprendo su trascendencia y tomo partido claramente por considerar que la educación universitaria debe ser un bien público, un derecho humano y que debe ser garantizada por el Estado, siguiendo la declaración de la Conferencia Regional sobre Educación Superior de Cartagena en 2008, (no retomada en París en la reunión mundial). Estos postulados deben ser, en mi opinión, la política pública de nuestros estados y países, aunque parece que soplan otros vientos...

2.- Respecto de la categoría tan en uso de POLÍTICA PÚBLICA, pasaré por alto la tentación de discutir el sintagma "políticas públicas". Solo mencionaré que asistimos a una puja muy ardua en América Latina en torno a qué política es la que mejor garantiza el derecho a la salud de nuestras poblaciones. En mi país, Argentina, puede verse en la página del Ministerio de Salud un llamado a la construcción del CUS (Cobertura Universal de Salud), política impulsada por el Banco Mundial y la Organización Panamericana de la Salud. El debate requiere una postura atenta y crítica, tanto en el terreno de la práctica como en el discurso. Entendemos que en el fragmentado campo de la financiación, los sistemas y servicios de salud de mi país enfrentan un proceso de desentendimiento del Estado y un avance de la privatización. En este contexto, la formación de la fuerza de trabajo ${ }^{1}$, es una arena de lucha permanente y particularmente sensible. La defensa y/o propuesta de una universidad de ingreso irrestricto, gratuita, autónoma, cogobernada y con currículas que en su forma y fondo se correspondan con los intereses del bien común -y que esto sea una Política Pública- será el centro de nuestra posición, que trataremos de explicitar.

Tal como lo menciona Juan Cesar García durante la Conferencia Regional de Educación Superior en América Latina y el Caribe, en 2008: "La Educación Superior es un bien público social, un derecho humano y universal y un deber del Estado. Esta es la convicción y la base para el papel estratégico que debe jugar en los procesos de desarrollo sustentable de los países de la región...”

3.- Centrémonos en la cuestión de la FORMACIÓN DE TRABAJADORES EN EL ÁREA DE SALUD EN AMÉRICA LATINA. Lo haremos desde la perspectiva Médico-Social: Para el movimiento de la medicina social latinoamericana, esta cuestión constituye un punto fundante de su agenda de investigación y acción. Recordemos que la Asociación Latinoamericana de Medicina Social (ALAMES) se funda en Ouro Preto, bajo el fuerte impacto del fallecimiento de Juan César García, médico pediatra argentino formado como sociólogo en Chile, y que desde el área de Recursos Humanos de la OPS, en Washington, realizará una colosal labor promoviendo el uso de las Ciencias Sociales como instrumento analizador del campo de la salud, estando algunos de sus aportes centrados en la formación de profesionales y trabajadores de la salud. La Salud Colectiva nace en Brasil, hermanada con la Medicina Social, con una intensa interacción en el ámbito de la producción académica, la solidaridad y la generación de iniciativas político-sanitarias.

Es justamente el punto de la formación de profesionales y trabajadores del campo sanitario una arena de lucha de concepciones y prácticas con repercusión intensa en el curso de los acontecimientos y en la conformación de las correlaciones de fuerza en nuestros países.

1 Horrorosamente llamada "Recursos Humanos"; aunque peor aún es el concepto de Capital Humano, de los economistas de Chicago -Gary Becker y otros-. 
La importancia del pensamiento de García puede recogerse en el trabajo "El pensamiento social en salud en Latinoamérica: releyendo a Juan César García”, escrito por Everardo Duarte Nunes y editado por la Universidade Estadual de Campinas:

“... Todos esses trabalhos abrem uma ampla discussão sobre o ensino, a busca de paradigmas para as ciências sociais (às vezes muito próximos dos modelos médicos) que pudessem atrair o interesse dos alunos para os aspectos sociais e toda uma discussão sobre a formação de recursos humanos em saúde que culminaria com o livro La Educación Médica en la América Latina. Antes, García, usando o tempo como variável, elabora uma tipologia das escolas médicas em: inovadoras, as que respondem rapidamente às novas ideias, as que respondem mais lentamente, e as procrastinadoras ou resistentes às mudanças...”

4.- Parece imperativo efectuar un punteo de los momentos más relevantes en la formación de los trabajadores del campo salud y de los médicos en particular; pero también en el conjunto de profesiones y saberes que se involucran en el campo de la salud. Comencemos por Abraham Flexner, figura clave a la hora de pensar contra qué reaccionamos y a qué nos enfrentamos. Diremos que el Modelo Médico Hegemónico (MMH), biologicista, ahistórico, asocial, autoritario, mercantilista, etc. descripto por Eduardo Menéndez y otros científicos sociales, tiene como hecho originario la influencia de Flexner. Por encargo de la Rockefeller Fundation y la Universidad de Hopkins, Flexner da inicio a una reforma de la educación médica, modificando contenidos y métodos cuyo alcance inicial son los EEUU y Canadá, pero que luego se extiende al mundo, dando origen a lo que conocemos como "medicina flexneriana”. Entre sus resultados cuenta con el hecho de haber estandarizado la formación de médicos en escala planetaria. La idea de iniciar la formación de medicina y otras carreras del campo salud con conocimientos de matemática, física y química, el estudio de la fisiología y de la clínica, así como el estudio de órganos aislados, ha conformado un profesional médico crecientemente funcional al mercado fármaco-tecnológico actual. Los médicos resultan así estandarizados; se trata de otra de las tantas consecuencias de la época de la estandarización como forma de organización del trabajo, la participación de la "Rockefeller - Standard Oil" no resulta así ajena al proceso. De hecho, los médicos nos parecemos en todo el planeta.

5.- En Latinoamérica destacaremos la influencia de la REFORMA UNIVERSITARIA DE CÓRDOBA en 1918, acontecimiento próximo a cumplir 100 años. Este hecho histórico tiene clara influencia en el perfil de nuestros profesionales universitarios latinoamericanos hispanoparlantes -Brasil desarrolla sus universidades con posterioridad-. Los acontecimientos de Córdoba en 1918 conducen a una universidad autónoma, cogobernada, con libertad de cátedra, fuertemente influida por la reciente Revolución Rusa, que significó un soplo de aire fresco para las concepciones académicas latinoamericanas. Se extendieron las luchas estudiantiles y los subsiguientes cambios en la currícula y el clima organizacional en las instituciones de enseñanza superior en América Latina. En el caso de Argentina, la Reforma del 18 se potencia con la instauración del ingreso irrestricto y la gratuidad instituida por el primer gobierno peronista (1946 - 1952), que facilitan la incorporación de importantes masas de jóvenes, hijos de familias de sectores medios y de trabajadores, a la vida universitaria. Esta conquista, que con sus altibajos se prolonga, resultó fuertemente expandida en el período 2003 -2015 y hoy es el motivo de las luchas de estudiantes y profesores en Argentina. En los últimos 50 años, mi país pasó de 9 universidades nacionales con carrera de medicina a 54, multiplicándose por seis. 
6.-EL MÉDICO DE FAMILIA CUBANO. La influencia de la revolución cubana en el terreno sanitario es clave, sobre todo a partir de la propuesta del propio Fidel de generar un médico cada 120 familias. Resultaría impensable haber logrado cobertura de este modelo para toda la isla sin el enorme esfuerzo realizado por la universidad cubana. En 10 años Cuba contó con médicos con el perfil y en número suficiente para llevar a cabo este proyecto, convirtiéndose en una pieza clave de la legitimidad del proceso revolucionario y una pieza maestra de las relaciones internacionales de Cuba. Asimismo, es grande la influencia médica cubana en múltiples ámbitos internacionales, como por ejemplo el programa BARRIO ADENTRO, en Venezuela, que brindó acceso a la atención sanitaria a amplios sectores de la población excluida de asistencia. También se destaca la Universidad Bolivariana; su creación se dio en el marco de la resistencia de la Universidad Central de Venezuela a formar profesionales con el perfil del médico de familia. Es importante mencionar la labor de la ELAM (Escuela Latino Americana de Medicina - Cuba), y en Argentina ya son más de 1200 los médicos formados en la ELAM e incorporados a nuestros servicios de salud.

7.- Otro influyente ámbito desde el que se impulsan nuevos criterios de formación de profesionales es el área de salud de la UAM-Xochimilco, donde se experimenta un clima de innovación en la enseñanza, incorporándose el enfoque de educación modular y comunitaria. Tras los hechos de Tlatelolco en 1968, y en medio de fuertes reclamos estudiantiles por vincular sus estudios a las necesidades populares aparece, sobretodo en la sede Xochimilco de la UAM, lugar donde se cursan carreras muy innovadoras en el campo sanitario y con gran influencia del pensamiento desarrollado en la Maestría de Medicina Social (en la que trabajan, entre otros, Catalina Eibenschutz, José Blanco Gil y Asa Cristina Laurell). La influencia de la UAM sigue muy vigente. Lógicamente, mencionado el proceso mexicano del 68, corresponde recordar que con cierta simultaneidad se produce el MAYO FRANCÉS, con una gran potencia revulsiva e influencia en los ámbitos universitarios en escala planetaria.

8.- Un elemento que citaré brevemente, pero gigante en su influencia, es la enseñanza de Paulo Freire sobre la educación como búsqueda y práctica de libertad. Se me permitirá decir que a ese pensamiento imprescindible se puede sumar una pléyade de pensadores, entre los que destacaré a María Isabel Rodríguez, en el área de RRHH de la OPS - Washington y otros ámbitos de trabajo, consecuente con la obra de Juan César, ejemplo de lucha, animadora de todas las iniciativas latinoamericanas innovadoras en Educación Médica, y que continúa siendo una referente en nuestros países y en su país, El Salvador, donde, tras ser Rectora y Ministra, hoy es asesora del presidente, Salvador Sánchez Cerén.

9.- Cabe resaltar la influencia de los organismos internacionales y las iniciativas de APS y SALUD PARA TODOS. Estas iniciativas desencadenan fuertes cambios conceptuales en el desarrollo formativo de profesionales en el campo de la salud. La estrategia de APS lanzada por la OMS en Alma Ata, y su posterior meta de salud para todos en el año 2000, modeló la fuerza laboral necesaria para ese proyecto. En el mismo sentido, operó otro dispositivo asociado a la categoría "promoción”, que tiene como hito más relevante la Carta de Ottawa. Promoción y Prevención pasan a ser referencia curricular de muchos emprendimientos, en particular en las carreras orientadas a medicina general o familiar.

10.- Corresponde evaluar qué nuevos paradigmas originados en el PSICOANÁLISIS, las investigaciones sobre GÉNERO, sobre el colectivo Lesbianas, Gays, Bisexuales y Transexuales 
(LGTB), así como la irrupción de actores políticos centrados en los PUEBLOS ORIGINARIOS (INTERCULTURALIDAD - BUEN VIVIR), han crecido en influencia en el campo de la formación de la fuerza de trabajo. Vienen ganando importancia categorías que provienen de los dispositivos científicos que están abordando los cambios en la concepción de nuevos paradigmas de identidad, sexualidad, familia, etc. Estas cuestiones, que han ganado su lugar en la cuestión social, interpelan y potencialmente cambian las formas tradicionales y cristalizadas de formar a los trabajadores de la salud.

11.- Múltiples EXPERIENCIAS LATINOAMERICANAS vienen dando cuenta tanto de situaciones críticas como de los esfuerzos y estrategias de los actores para superar los paradigmas conservadores, para generar políticas públicas comprometidas con el derecho a la salud. Esto se expresa en el terreno de la vida universitaria de diversos modos. Mencionaremos:

1. LAS LUCHAS DE CHILE: Contra un modelo educativo privatista que se ha hecho parte de la cadena de negocios crediticios bancarios y que modela y condiciona sus perfiles. Hoy en debate generado por la luchas estudiantiles y de profesores.

2. LA ELAM EN CUBA: Con su labor en la formación de médicos comprometidos con el pueblo es el único país en el mundo que exporta médicos. Profesionales que están donde los médicos egresados del Modelo Flexneriano no quieren estar.

3. BRASIL Y LOS LÍMITES ENCONTRADOS POR EL SISTEMA ÚNICO DE SALUD, SUS: Fue necesario generar el programa MAIS MÉDICOS para brindar cobertura a sectores poblacionales desfavorecidos y poner de manifiesto el rol de la "escasez de médicos" y de la falta de acompañamiento a las necesidades de la población por parte de la universidad, así como el rol del arancelamiento. Este fenómeno explicaría la necesidad de recurrir a médicos extranjeros. Los límites de las medidas progresivas registradas en favor de la equidad de raza, etc., pueden expresarse en la presencia de numerosos contingentes de estudiantes brasileños en las universidades argentinas. El Decano de la Facultad de Medicina de la Universidad de Rosario mencionó públicamente que cree tener evidencia que Medicina de Rosario es la cuarta facultad pública con más estudiantes de medicina brasileños, la segunda de haitianos y la tercera con más colombianos.

4. La VENEZUELA de Hugo Chávez se encontró con graves conflictos para generar programas de extensión de cobertura médica, como lo es BARRIO ADENTRO. La respuesta fue apelar a la masiva cooperación cubana y a la apertura de nuevas escuelas de medicina.

5. Las dificultades de acceso a la Universidad en MÉXICO ha generado malestar entre los jóvenes. El partido Morena responde generando iniciativas de oferta universitaria auto gestionada.

12.- NUESTRA EXPERIENCIA UNLAM (Universidad Nacional de La Matanza), TRIUNFO CONCEPTUAL. He tenido el alto honor y el placer de haber participado desde el inicio (2011) de una experiencia que ha marcado mi reflexión en torno a la formación de la fuerza de trabajo en salud. Me refiero a la formulación y puesta en práctica durante cuatro años de la carrera de medicina en la UNLAM, y luego dos años dolorosos de resistencia a su desmantelamiento. El Profesor Mario Rovere fue convocado por la UNLAM para ser Decano del Departamento de 
Salud y organizador de la carrera de Medicina, primera universidad pública que pone en marcha esta carrera en el conurbano bonaerense ${ }^{2}$.

La UNLAM ${ }^{3}$ nace en los noventa, fruto de la política del gobierno peronista y neoliberal (i!) del presidente Carlos Saúl Menem, constituyendo uno de los escasos efectos positivos de la época. El gobierno de los doce años post crisis del 2001, de Néstor Kirchner y Cristina Fernández de Kirchner, retoman el desarrollo de estas universidades, creando además nuevas casas de estudio en el conurbano y numerosas ciudades del interior. En el caso de la UNLAM, pasó de tener 8 mil estudiantes a 45 mil. En el 2010 se diseña la Carrera de Medicina.

Inicia su funcionamiento una carrera de Medicina, una carrera innovadora:

1. En su ideología, claramente comprometida con el derecho a la salud, basada explícitamente en el ideario de la Medicina Social Latinoamericana y con compromiso con los intereses populares.

2. En su práctica, carente de todo sadismo hacia los estudiantes, con claro compromiso de centrar la actividad del proceso enseñanza-aprendizaje en el polo del aprendizaje. Renunciando a las clases magistrales, centrando el aprendizaje en los estudiantes a través del dispositivo de aprendizaje basado en problemas (ABP).

3. Énfasis en las prácticas tempranas, con presencia en la comunidad desde el inicio de la carrera, generando Habilidades Clínicas y concibiendo siempre la temática de salud ampliada como cuestión extendida a los procesos de determinación social.

4. Con técnicas y prácticas de conducción de la enseñanza en comisiones con un tutor cada 10 estudiantes, con espacio de practicum reflexivo tras cada práctica.

5. La evaluación en proceso, con docentes que realmente conocen y ayudan en el proceso de aprendizaje.

La reacción conservadora no se hizo esperar; cuando percibieron que la tarea fundante de nuestra institución tomaba vuelo, se procedió a:

El apartamiento del Decano, Dr. Mario Rovere, cuya experiencia en la temática de Educación Médica y en planificación estratégica de los recursos humanos resultó imprescindible para la formulación y el desarrollo del proyecto. Rovere supo conducir el proceso de manera participativa, democrática y en torno a su labor se movilizó un equipo tanto médico como educativo y de ciencias sociales muy calificado. Además, jugó un papel indispensable en la convocatoria del equipo de profesionales que acompañó el proyecto de innovación en la educación médica.

Lo segundo que ocurrió fue el despido de docentes "díscolos", que manifestaron su contrariedad por lo relatado.

Posteriormente se produjo el cese de un conjunto de 30 profesores de más de 70 años y profesoras de más de 65 años, que fueron fundamentales por su experiencia. Estos compañeros

2 El conurbano: se ha descripto a la Argentina como un país deforme con una gran cabeza. Un interior poco poblado y pobre y una cabeza, Buenos Aires, puerto de entrada de mercancías manufacturadas y salida de nuestros productos (en general de origen agrario). Lo cierto es que la Ciudad Autónoma de Buenos Aires, antes Capital Federal, no ha variado en el último siglo su número de pobladores, unos 3 millones. El conurbano bonaerense, en cambio, ha crecido, constituyendo una megalópolis de unos 12 millones de habitantes, siendo la población de toda la provincia de Buenos Aires asiento de más del 40\% de la población argentina (unos 43 millones en la actualidad). El partido de La Matanza es el más populoso, con unos 2 millones de habitantes.

3 La Matanza: por la concentración demográfica mencionada, por ser asiento de industrias manufactureras y de numerosas "villas de emergencia" (barriadas pobres con escasa y a veces nula infraestructura urbana), es asiento de la conflictiva social más virulenta. 
fueron notificados por correo electrónico, sin agradecimiento alguno por sus aportes como profesionales híper calificados y comprometidos. Entre los despedidos está el Profesor Víctor Penchaszadeh, único argentino que participó en la investigación que llevó a la decodificación del genoma humano, que además fue un perseguido político que tuvo que exiliarse en Caracas y Nueva York durante la dictadura cívico-militar (1976-1983), donde se destacó en su labor como genetista y bioeticista en la New York University. Es además el principal motor científico que, a partir de las demandas de Abuelas de Plaza de Mayo, llevó a la comunidad científica la problemática de sus nietos expropiados durante la última dictadura argentina. El buen uso de la ciencia concluye en la concreción del "índice de abuelidad", que nos permite identificar a los jóvenes privados de su identidad por la dictadura. Mientras Víctor es condecorado en otros países, aquí fue apartado sin explicaciones -a través de un correo-e- de la carrera a la que tanto aportó. Para muchos resultó algo imperdonable.

Fue por estas causas que formamos un Sindicato: Sindicato de Investigadores y Docentes de la Universidad de La Matanza. A fin del año 2016 fueron despedidos todos los docentes que integraban la Comisión Directiva y algunos más, a pesar de tratarse de Derechos protegidos por la Constitución Nacional. En total fueron más de 20 trabajadores.

Declaramos una huelga pidiendo la reincorporación de dichos trabajadores. A fines de abril de 2017 fueron desvinculados gran número de huelguistas. Hasta el momento son más de 100 despedidos. La carrera se ha degradado y los estudiantes sufrieron la ruptura de un contrato explícito.

Mario Rovere, en su carta de renuncia dice: “... En 43 años de profesión y en más de 30 años de consultor de desarrollo de Recursos Humanos en Salud, recorriendo y cooperando con instituciones educativas de 20 países y casi todas las universidades públicas del nuestro, nunca conocí un "academicidio" de esta magnitud. Nunca vi, ni escuché (al menos en democracia) que una Carrera de Medicina, que aún no ha graduado su primera promoción, haya "desechado” en un año y medio a 100 profesores (entre cesantías, jubilaciones anticipadas y renuncias bajo coacción). Cien profesores, a quienes antes se les brindó una capacitación intensiva y focalizada en este tipo de currícula y de quienes no consta una sola observación formal o escrita sobre su mal desempeño laboral y/o académico..."

Los estudiantes nos han acompañado con limitaciones, pues ellos no quieren malograr lo conquistado. Nos consta su desconsuelo. Se tratará de una experiencia inolvidable.

13.- ESTA FILOSOFÍA DE CAMBIO CURRICULAR y de compromiso social en una carrera de Medicina, vive e interpela. Hoy está viva en facultades de Medicina como la de ROSARIO, que es una carrera muy antigua, con un proceso de innovación curricular muy intenso, con estudiantes masivos a favor del ingreso irrestricto y su gratuidad. También en Bahía Blanca, experiencia que comenzó con anterioridad a desarrollar una carrera de medicina innovada, que además aportó conocimientos y experiencias inestimables a través de los cursos de Innovación en Educación Médica, preparatorios de los docentes de la experiencia UNLAM. También en la Universidad JAURETCHE, la Universidad del CENTRO, y la universidad de MAR DEL PLATA, que inicia su curso de Medicina en 2017, con modalidad afín y que además tiene ingreso irrestricto.

$1^{\circ}$ CONCLUSIÓN: Todo hace pensar que existe un gran movimiento, que es continental, fuertemente fundamentado y con raigambre histórica en el reclamo de acceso masivo a la educación universitaria, lo cual a veces es necesario para sostener los proyectos de construir un sistema único 
de salud, como serían los casos de Brasil o El Salvador; o como ocurre en Argentina, como estrategia para incidir en el cuestionamiento y paliar la inequidad y mercantilización del sector salud.

$2^{\circ}$ CONCLUSIÓN: El malestar y la CRISIS DE LA ENSEÑANZA es muy aguda en las Facultades de Medicina y otras profesiones de la salud latinoamericanas. Esto requiere introducir cambios sustantivos en sus currículas, que las adapten al imperativo ético de completar los ciclos de construcción de ciudadanía, de contar con servicios nacionales integrales de salud, financiados desde rentas generales, con participación y control popular, que brinden servicios de calidad, oportunos, resolutivos, humanizados, fuera de las crueles características del mercado. Democracia y salud deben tornarse sinónimos.

Bibliografía

- Flexner A. Medical Education in the United States and Canada. A Report to the Carnegie Foundation for the advancement of Teaching. Bulletin. No.4. Boston, Massachusetts: Updyke; 1910. 